\title{
BRYOPHYLLUM (CRASSULACEAE): ESPECIES ORNAMENTALES NATURALIZADAS EN LA ARGENTINA
}

\author{
JULIO A. HURRELL ${ }^{1}$, GUSTAVO DELUCCHI², HÉCTOR A. KELLER ${ }^{3}$, PABLO C. STAMPELLA \& ELIÁN L. \\ GUERRERO 5
}

\begin{abstract}
Resumen: Hurrell, J.A., G. Delucchi, H. A. Keller, P.C. Stampella \& E.L. Guerrero. 2012. Bryophyllum (Crassulaceae): especies ornamentales naturalizadas en la Argentina. Bonplandia 21(2): 169-181.

Este trabajo incluye cuatro especies ornamentales de Bryophyllum (Crassulaceae): $B$. daigremontianum (Raym.-Hamet \& H.Perrier) A.Berger, B. delagoense (Eckl. \& Zeyh.) Schinz, B. fedtschenkoi (Raym.-Hamet \& H.Perrier) Lauz.-March. y B. pinnatum (Lam.) Oken, naturalizadas en la Argentina. Las tres primeras son nuevas citas para el país; para B. pinnatum, citada con anterioridad, se amplía su área. Se incluye una clave para la identificación de las especies, descripciones, distribución, datos etnobotánicos, observaciones, material de referencia y comentarios sobre su naturalización.
\end{abstract}

Palabras clave: Bryophyllum, flora adventicia, Argentina, ornamentales, naturalización.

Summary: Hurrell, J.A., G. Delucchi, H. A. Keller, P.C. Stampella \& E.L. Guerrero. 2012. Bryophyllum (Crassulaceae): ornamental species naturalized in Argentina. Bonplandia 21(2): 169-181.

This paper includes four ornamental species of the genus Bryophyllum (Crassulaceae): $B$. daigremontianum (Raym.-Hamet \& H.Perrier) A.Berger, B. delagoense (Eckl. \& Zeyh.) Schinz, B. fedtschenkoi (Raym.-Hamet \& H.Perrier) Lauz.-March. and B. pinnatum (Lam.) Oken, all naturalized in Argentina. The first three are new records for this country; for $B$. pinnatum, cited previously, its area is extended. Also includes an identification key, descriptions, distribution, ethnobotanical data, reference material and comments on their naturalization.

Key words: Bryophyllum, adventitious flora, Argentina, ornamentals, naturalization.

\section{Introducción}

Este trabajo incluye cuatro especies del género Bryophyllum Salisb. (Crassulaceae), naturalizadas en la Argentina; tres de ellas se citan por primera vez para nuestro país: $B$. daigremontianum (Raym.-Hamet \& H.Perrier) A.Berger (=Kalanchoe daigremontiana Raym.-

${ }^{1}$ Laboratorio de Etnobotánica y Botánica Aplicada. Facultad de Ciencias Naturales y Museo, Universidad Nacional de La Plata, Calle 64 nro. 3, 1900-La Plata, Argentina. Investigador CONICET. E-mail: juliohurrell@gmail.com

${ }^{2}$ Facultad de Ciencias Naturales y Museo, Universidad Nacional de La Plata, Paseo del Bosque s.nro., 1900-La Plata, Argentina. E-mail: delucchi@fcnym.unlp.edu.ar

${ }^{3}$ Facultad de Ciencias Forestales, Universidad Nacional de Misiones - Instituto de Botánica del Nordeste, Casilla de Correo 209, 3400-Corrientes, Argentina. E-mail: hakeller2000@yahoo.com.ar

${ }^{4}$ Laboratorio de Etnobotánica y Botánica Aplicada. Facultad de Ciencias Naturales y Museo, Universidad Nacional de La Plata, Calle 64 nro. 3, 1900-La Plata, Argentina. Becario UNLP. E-mail: pstampella@yahoo.com

${ }^{5}$ Instituto Fitotécnico de Santa Catalina, Facultad de Ciencias Agrarias y Forestales, Universidad Nacional de La Plata. Garibaldi 3400, 1836-Llavallol, Argentina. E-mail: elianrma@yahoo.com.ar 
Hamet \& H.Perrier), B. delagoense (Eckl. \& Zeyh.) Schinz (=K. delagoensis Eckl. \& Zeyh.), B. fedtschenkoi (Raym.-Hamet \& H.Perrier) Lauz.-March. (=K. fedtschenkoi Raym.-Hamet \& H.Perrier); la cuarta especie, B. pinnatum (Lam.) Oken (=K. pinnata Lam.), había sido mencionada con anterioridad para Corrientes (Martínez Crovetto, 1981), y se amplían sus registros para Misiones. Se presenta una clave para la diferenciación de las especies, sinonimias, descripciones, distribución geográfica, nombres vernáculos más frecuentes, datos etnobotánicos, observaciones, material de referencia y comentarios sobre su naturalización.

Estas especies fueron introducidas en la Argentina con fines ornamentales, al igual que en otras partes del mundo, donde asimismo se han naturalizado $\mathrm{y}$, en algunos casos, devienen malezas o invasoras. En este sentido, su primer registro en el país resulta de interés para evaluar su comportamiento futuro, en el marco de las invasiones biológicas.

La familia Crassulaceae, con unos 35 géneros y ca. 1500 especies, presenta amplia distribución mundial, con centros de diversificación en México y Sudáfrica, y poco representada en Sudamérica austral y Australia (Eggli, 2003; Thiede \& Eggli, 2007; Walters $\&$ al., 2011). En la Argentina se han registrado 8 especies nativas de Crassula L.; 2 nativas y 1 adventicia de Sedum L.; 1 especie nativa de Echeveria DC., y la adventicia $B$. pinnatum (sub Kalanchoe pinnata); en total, 13 especies (Freire-Fierro, 2008). Con las nuevas especies de Bryophyllum aquí citadas, el género está representado en nuestra flora por 4 especies, y el total de las Crassulaceae asciende a 16 especies.

\section{Materiales y Métodos}

Esta contribución se basa en diversos viajes de estudio, en los que se coleccionó material de herbario, depositado para su documentación en los herbarios CTES (Instituto de Botánica del Nordeste, Corrientes) y LP (Plantas Vasculares, Museo de La Plata). Se consultaron, asimismo, distintos herbarios y bibliotecas.

Con el fin de recabar datos sobre los usos, el cultivo y origen de la naturalización de las especies tratadas, se realizaron entrevistas etnobotánicas abiertas a distintos pobladores locales (Albuquerque \& Lucena, 2004; Martin, 2004). Los relevamientos etnobotánicos, como ocurre con los estudios ecológicos sobre cambios ambientales (Lane, 1997), complementan los registros históricos y florísticos, con miras a la comprensión de los procesos involucrados en la naturalización de las especies exóticas introducidas.

\section{Resultados}

Bryophyllum Salisb., Parad. Lond. p1. 3, 1805.

Hierbas perennes o bienales, suculentas, glabras. Hojas persistentes, mayormente opuestas y decusadas, simples o pinnadas, en general pecioladas; láminas planas o cilíndricas, con propágulos en márgenes y ápices. Inflorescencias terminales, cimosas, a veces con propágulos. Flores actinomorfas, bisexuales, 4-meras, péndulas. Cálizpersistente, tubuloso a urceolado, más corto que la corola, a veces dilatado en la base, acrescente, lobos deltoides. Corola gamopétala, tubulosa a infundibuliforme; tubo más largo que los lobos, a veces contraída en la base, lobos en general obovados. Estambres 8, en 2 ciclos, insertos en la base del tubo de la corola o por debajo de su mitad, incluidos o exertos. Carpelos libres; estilos más largos que el ovario. Nectarios 4, libres. Folículos incluidos en el cáliz. Semillas obovoides o elipsoides, estriadas o rugosas.

Distribución geográfica. Género endémico de Madagascar, con 20-30 especies; algunas introducidas con fines ornamentales $y$ naturalizadas en diversas partes del mundo $(\mathrm{Fu}$ \& Gilbert, 2001; Moran, 2009; Walters \& al., 2011).

Reproducción y multiplicación. La floración es invernal; a veces, también primaveral. La polinización es básicamente ornitófila. Las semillas son barocoras y anemocoras (Thiede \& Eggli, 2007). Sin embargo, la forma más frecuente de expansión y de naturalización es 
por vía vegetativa, a través de los propágulos o brotes adventicios, a veces llamados "hijuelos" o "mudas", desarrollados en los márgenes de las hojas, cuya morfogénesis ha sido estudiada (Parsley \& Rushing, 2003; Garcés \& al., 2007; Garcés \& Sinha, 2009). A esta característica distintiva hace referencia el nombre genérico: del griego bryon ( $\beta \rho v o v)$, 'brote', y phyllon (

Observación. Si bien Bryophyllum se ha considerado una sección de Kalanchoe Adans., de distribución más amplia en África y Asia tropical (Thiede \& Eggli, 2007; Chernetskyy, 2011, 2012), aquí se lo trata de forma separada, según la opinión de distintos autores $(\mathrm{Fu} \&$ Gilbert, 2001; Moran, 2009; Walters \& al., 2011). Estos géneros se diferencian por los siguientes caracteres:

1. Flores péndulas. Sépalos unidos en la base o formando un tubo. Estilos más largos que los ovarios. Hojas simples o imparipinnadas, con propágulos (plantas “vivíparas”).

Bryophyllum

1'. Flores erectas. Sépalos unidos en la base. Estilos más cortos que los ovarios. Hojas simples, sin propágulos (plantas no "vivíparas”).

Kalanchoe

\section{Clave de las especies de Bryophyllum naturalizadas en la Argentina}

1. Hojas planas, pecioladas, con propágulos en sus márgenes. Hierbas perennes.

2. Hojas simples. Tubo del cáliz hasta $2 \mathrm{~cm}$ long. Corola hasta $3 \mathrm{~cm}$ long. Estambres ligeramente exertos. Carpelos hasta $1 \mathrm{~cm}$ long. Nectarios hasta $1 \mathrm{~mm}$ long.

3. Plantas hasta 2,5 m alt., tallos robustos. Cimas paniculadas laxas; ramas hasta $15 \mathrm{~cm}$ long. Tubo del cáliz 3-4 mm long. Carpelos 4-5 mm long., estilos 1,1-1,4 cm long.

1. B. daigremontianum

3'. Plantas hasta $80 \mathrm{~cm}$ alt., tallos delgados. Cimas corimbiformes densas, ramas hasta $5 \mathrm{~cm}$ long. Tubo del cáliz 6-14 mm long. Carpelos 0,8-1 cm long., estilos 1,4-1,5 cm long.

2. B. fedtschenkoi

2'. Hojas pinnadas, excepto las primeras, que son simples. Tubo del cáliz 2-4 cm long. Corola 3-7 cm long. Estambres incluidos. Carpelos 1,2-1,4 cm long. Nectarios 1,8-2,6 mm long.

3. B. pinnatum

1'. Hojas cilíndricas, sésiles o con pecíolo indistinguible, con propágulos hacia el ápice. Hierbas bienales o perennes.

4. B. delagoense

1. Bryophyllum daigremontianum (Raym.Hamet \& H. Perrier) A.Berger, Nat. Pflanzenfam. (ed. 2), 18a: 411. 1930 (Figs. 1, 2 A-B).

Kalanchoe daigremontiana Raym.-Hamet \& H. Perrier, Ann. Mus. Colon. Marseille, sér. 2, 2: 128132. 1914.

Hierbas perennes, 0,5-2,5 $\mathrm{m}$ alt. Tallos robustos, erectos o decumbentes, teretes, por lo común no ramificados. Hojas opuestas, simples, carnosas; pecíolo subterete, 1-5 cm long.; láminas deltoides a elípticooblongas, 5-25 cm long. × $3-12 \mathrm{~cm}$ lat., glaucas, haz verde, envés grisáceo o verdoso con manchas purpúreas, ápice agudo, base cuneada o truncada, margen crenado-serrado, con propágulos en las muescas. Cimas paniculadas laxas, $15-30 \mathrm{~cm}$ diám.; ramas hasta $15 \mathrm{~cm}$ long.; pedicelos 0,5-1,5 cm long. Cáliz verde o purpúreo; tubo 3-4 mm long.; lobos 3-7 mm long., agudos. Corola 2-3 cm long., rosada, rojiza o purpúrea; lobos 6-12 mm long. $\times 3,5-4,5 \mathrm{~mm}$ lat., agudos. Estambres poco exertos. Carpelos 4-5 mm 

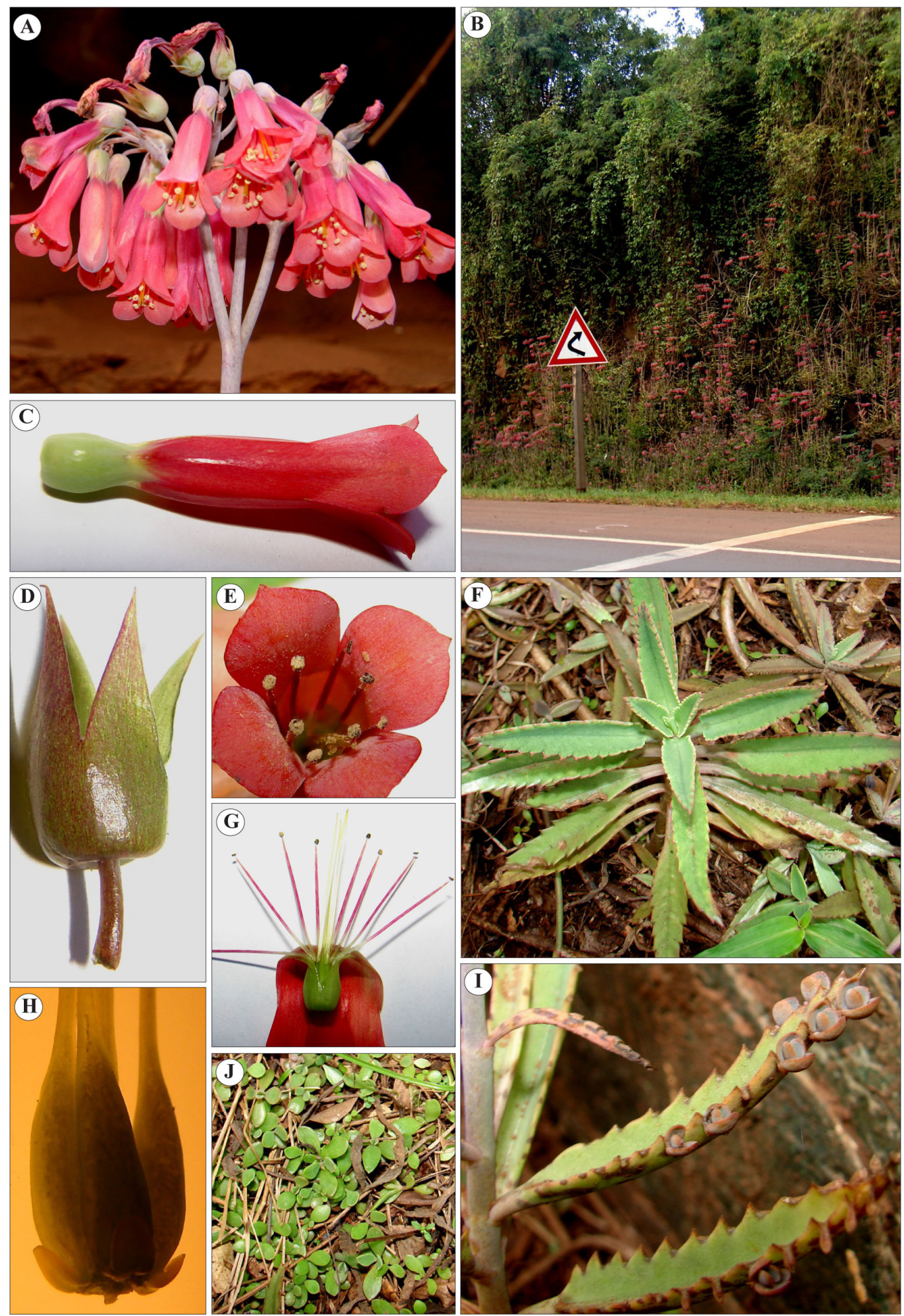

Fig. 1. Bryophyllum daigremontianum. A: Inflorescencia. B: Población en Piray Guazú, Misiones. C: Flor. D: Cáliz. E: Lobos de la corola. F: Hojas. G: Detalle del pistilo y estambres. H: Carpelos y nectarios. I: Propágulos foliares. J: Desarrollo de propágulos (Fotos H.A. Keller). 
long.; estilos 11-14 mm long. Nectarios 0,6$1 \mathrm{~mm}$ long. Semillas $0,6-1 \mathrm{~mm}$ long. $2 n=38$ (Moran, 2009).

Distribución geográfica. Originaria de Madagascar, naturalizada en el sur de Europa, África y Asia, Hawái, Australia, Nueva Zelanda, sur de los Estados Unidos, Antillas, México, Nicaragua, Colombia, Ecuador y Bolivia. En los Estados Unidos y en Australia ha sido declarada maleza (Stevens \& al., 2001; Villaseñor \& EspinosaGarcía, 2004; Domingues \& Freitas, 2006; Guillot Ortíz \& al., 2009; Moran, 2009; Walters \& al., 2011; Randall, 2012). En la Argentina se cultiva (Dimitri, 1987) y crece naturalizada en las provincias de Misiones (Montecarlo, Candelaria) y Buenos Aires (Avellaneda, La Plata), y en la Ciudad Autónoma de Buenos Aires.

Nombres vernáculos: "calanchoe", "espinazo del diablo", "madre de miles", "mala madre", "planta de la fortuna".

Etnobotánica. Ornamental, muy difundida desde principios del siglo XX, una de las más cultivadas en la Argentina. En México, se utilizan las hojas, en emplastos, como antiinflamatorio (Estrada \& al., 2007). Se ha estudiado la actividad citotóxica y antimicrobiana de extractos de toda la planta (Nahar \& al., 2008).

Material examinado: ARGENTINA. Misiones. Montecarlo, Puente sobre arroyo Piray Guazú, 12-VII-2012, fl., Keller 10974 (CTES); Candelaria, Ruta Provincial 3, de Cerro Corá a Cerro Azul, 19-VI-2003, fl., Zuloaga \& al. 8067 (SI). Capital Federal. Avenida de Mayo y Salta, 30-VI-2012, fl., Delucchi 3538 (LP). Buenos Aires. Avellaneda. Sarandí, vías de ferrocarril, 31-XII-2011, fl., Guerrero 140 (LP). Wilde, Avenida Onsari 422, en balcón, 24-IX-2012, fl., Guerrero 231 (LP). Wilde, esquina de Mitre y Onsari, sobre techo de chapa, 24-IX-2012, fl, Guerrero 233 (LP). La Plata, La Plata, calle 69 (6 y 7), sobre viejo toldo metálico, 28-VIII2012, fl., Guerrero 223 (LP). La Plata, La Plata, avenida 7 (57 y 58), sobre cartel, 1-XI-2012 fl., Guerrero 289 (LP).
2. Bryophyllum fedtschenkoi (Raym.-Hamet \& H. Perrier) Lauz.-March., Compt. Rend. Hebd. Séances Acad. Sci. 278: 2508. 1974 (Fig. 2 C-F).

Kalanchoe fedtschenkoi Raym.-Hamet \& H.Perrier, Ann. Mus. Colon. Marseille, sér. 2, 3: 75-80. 1915.

Hierbas perennes, hasta $80 \mathrm{~cm}$ alt. Tallos delgados, erectos a postrados, a menudo radicantes, teretes, ramificados. Hojas opuestas, simples, carnosas; pecíolo terete, 1-6 mm long.; láminas obovadas o suborbiculares, $1-5(-10) \mathrm{cm}$ long. $\times 0,5-3(-8) \mathrm{cm}$ lat., a menudo grisáceo-purpúreas o grisáceolavanda, cerosas, ápice obtuso o redondeado, base cuneada, margen crenado, a veces entero hacia la base, con propágulos en las muescas. Cimas corimbiformes densas, hasta $20 \mathrm{~cm}$ diám.; ramas hasta $5 \mathrm{~cm}$ long.; pedicelos 0,5 $1,5 \mathrm{~cm}$ long. Cáliz verdoso a purpúreo; tubo 6-14 mm long.; lobos 4-7 mm long., agudos. Corola 1,8-2,5 cm long., anaranjado-rojiza, con rayas rojas; tubo 17-19 $\mathrm{mm}$ long.; lobos 5-8 mm long. $\times$ 4,2-4,6 mm lat., obtusos a redondeados. Estambres ligeramente exertos. Carpelos 0,8-1 cm long.; estilos 1,4-1,5 cm long. Nectarios 0,8-1 mm long. Semillas ca. 0,6 mm long. $2 n=34$ (Moran, 2009).

Distribución geográfica. Originaria de Madagascar, naturalizada en España, India, Sudáfrica, Hawái, Galápagos, Australia, sur de los Estados Unidos, México y las Antillas (Villaseñor \& Espinosa-García, 2004; Guillot Ortíz \& al., 2009; Moran, 2009; Walters \& al., 2011; Randall, 2012). En la Argentina se cultiva y ha sido hallada naturalizada en Salta (La Caldera), Misiones (Concepción de la Sierra), y en zonas urbanas de la provincia de Buenos Aires (Quilmes, La Plata).

Nombres vernáculos: "flor de carne chica" (Misiones), "calanchoe", "siempreviva".

Etnobotánica. Ornamental, con distintos cultivares (Eggli, 2003; Guillot Ortíz \& al., 2009; Walters \& al., 2011). En Misiones, sus flores rojizas son utilizadas por los niños para jugar a "la casita" o "la comidita", debido a que 

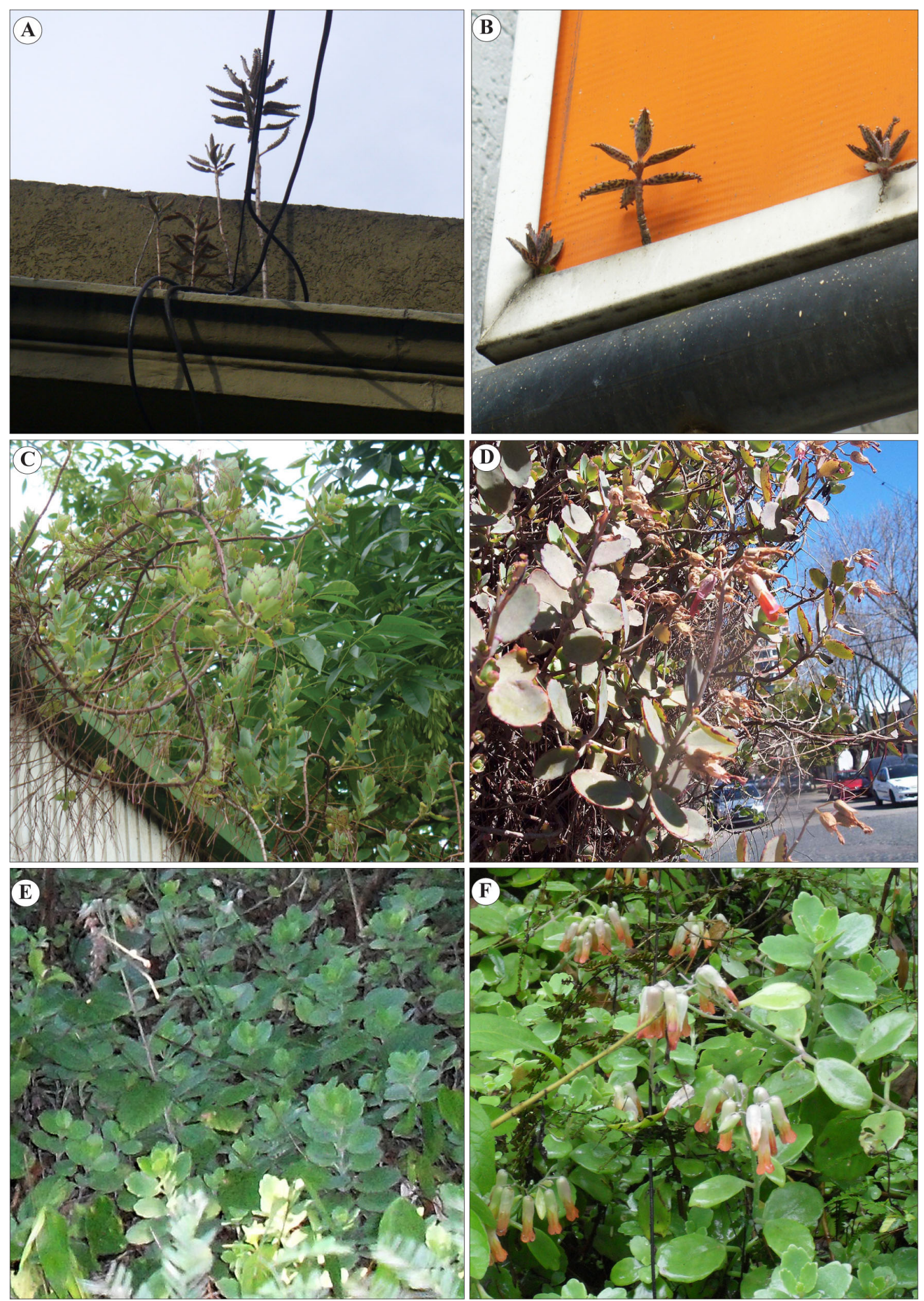

Fig. 2. Bryophyllum daigremontianum. A-B: Plantas sobre techos y carteles, en La Plata, Buenos Aires (Fotos: E. L. Guerrero). Bryophyllum fedtschenkoi. C: Plantas sobre un techo, en La Plata (Foto: E. L. Guerrero). D: Planta con flores en La Plata (Foto: G. Delucchi). E-F: Plantas en Concepción de la Sierra, Misiones (Fotos: P.C. Stampella). 
parecen "trozos de carne", de ahí su nombre local. Es una de las pocas plantas con flores rojizas disponibles en invierno.

Material examinado: ARGENTINA. Salta. La Caldera. Ruta Nacional 9, $24^{\circ} 30^{\prime} 22,9^{\prime \prime} \mathrm{S}-65^{\circ}$ 19' 1" W, borde de selva, 1420 m.s.n.m., 22-VII2009, fl., Keller et al. 7367 (CTES). Misiones. Concepción de la Sierra. Santa María, Paraje Cerro Mártires, chacra dos Santos, junto a B. pinnatum, pero más abundante, 18-VII-2012, fl., Stampella 154 (LP). Buenos Aires. Quilmes. Bernal, Cramer, entre Tacuarí y Ramella, en alero de comercio abandonado, 9-VII-2012, fl., Guerrero 209 (LP); Ramella, entre Roca y Carabelas, 29-IX-2012, fl., Guerrero 237 (LP). La Plata, ciudad de La Plata, calle 3 y 36, sobre árboles, 16-VIII-2007, fl., Delucchi 3118 (LP); 22-XI-2007, fl., Delucchi 3172 (LP); 27-VI-2012, fl, Delucchi 3537 (LP); 31-VIII2012, fl., Delucchi 3541 (LP).

3. Bryophyllum pinnatum (Lam.) Oken, Allg. Naturgesch. 3(3): 1966. 1841 (Fig. 3 A-B).

Cotyledon pinnata Lam., Encycl. 2(1): 141. 1786

Bryophyllum calycinum Salisb., Parad. Lond. pl. 3. 1805.

Kalanchoe pinnata (Lam.) Pers., Syn. Pl. 1: 446. 1805 .

Verea pinnata (Lam.) Spreng., Syst. Veg. 2: 260. 1825.

Hierbas perennes, 0,4-2 malt. Tallos robustos, erectos o ascendentes, teretes, simples o poco ramificados. Hojas opuestas, coriáceo-carnosas, las primeras simples, el resto imparipinnadas con 3-5 folíolos; pecíolos subteretes, 2-10 $\mathrm{cm}$ long.; hojas simples ovadas a elípticas, 5-20 cm long $\times 5-12 \mathrm{~cm}$ lat., verdes con vetas purpúreas, obtusas, base cuneada o truncada, margen crenado o doblemente crenado, con propágulos en las muescas; foliolos $6-20 \mathrm{~cm}$ long. $\times$ 4-12 cm lat., el terminal mayor. Cimas paniculadas laxas, $10-80 \mathrm{~cm}$ diám., ramas hasta $12 \mathrm{~cm}$ long.; pedicelos 1-2,5 cm long. Cáliz verdoso o amarillento con líneas rojizas o purpúreas, papiráceo luego de la antesis; tubo 2-4 cm long.; lobos 0,7-1,1 cm long., agudos o acuminados. Corola 3-7 cm long., roja o verde rojiza, tubo verdoso blancuzco oculto por el cáliz; lobos 9-20 mm long. × 4-6,5 mm lat., agudos o acuminados. Estambres incluidos. Carpelos 1,2-1,4 cm long.; estilos hasta $3 \mathrm{~cm}$ long. Nectarios 1,8-2,6 mm long. Semillas ca. 0,8 mm long. $2 n=40$ (Moran, 2009).

Distribución geográfica. Originaria de Madagascar, ampliamente naturalizada en zonas cálidas y templado-cálidas del mundo, como el sur de Europa, África, Pakistán, China, Australia, Nueva Zelanda, islas del Pacífico, sur de Estados Unidos, Mesoamérica, Antillas, Ecuador, Perú, Bolivia, norte y sudeste de Brasil; a menudo deviene maleza o invasora, sobre todo, en zonas tropicales $(\mathrm{Fu}$ \& Gilbert, 2001; Stevens \& al., 2001; Sarwar, 2004; Villaseñor \& Espinosa-García, 2004; Domingues \& Freitas, 2006; Pino Infante, 2006; Thiede \& Eggli, 2007; Moran, 2009; Walters \& al., 2011; Randall, 2012). En la Argentina se cultiva (Dimitri, 1987) y crece naturalizada en Corrientes (Martínez Crovetto, 1981); ha sido registrada, además, en Misiones (Concepción de la Sierra), por lo cual se amplía su área de distribución.

Nombres vernáculos: "mata callo", "maravilla" (Corrientes), "flor de carne grande" (Misiones), "calanchoe", "espíritu santo", "hoja del aire", "madre de millones", "maravillosa", "planta del milagro", "planta de la prosperidad", "siempreviva".

Etnobotánica. Ornamental (Walters \& al., 2011). En diversos países tanto del Viejo como del Nuevo Mundo se emplea en medicina popular como remedio antiséptico y antidermatósico, contra las infecciones de ojos y de oídos, resfríos, sinusitis, antitusivo, expectorante, febrífugo, antimalárico, antiepiléptico, digestivo, antidiarreico, hipotensor, depurativo, antinefrítico, antidiabético, anticáncer (Vega, 2001; Arango Caro, 2004; Tene \& al., 2007; GodínezCaraballo \& Volpato, 2008; Olowokudejo \& al., 2008; Jiofack \& al., 2010; Barukial \& Sarmah, 2011; Biswas \& al., 2011; Ghasi \& al., 2011; Chagas Nogueira \& al., 2012). Los niños de Polinesia Occidental ingieren el néctar (Whistler, 1988). En Perú, se utiliza como planta protectora contra la envidia (Pardo, 

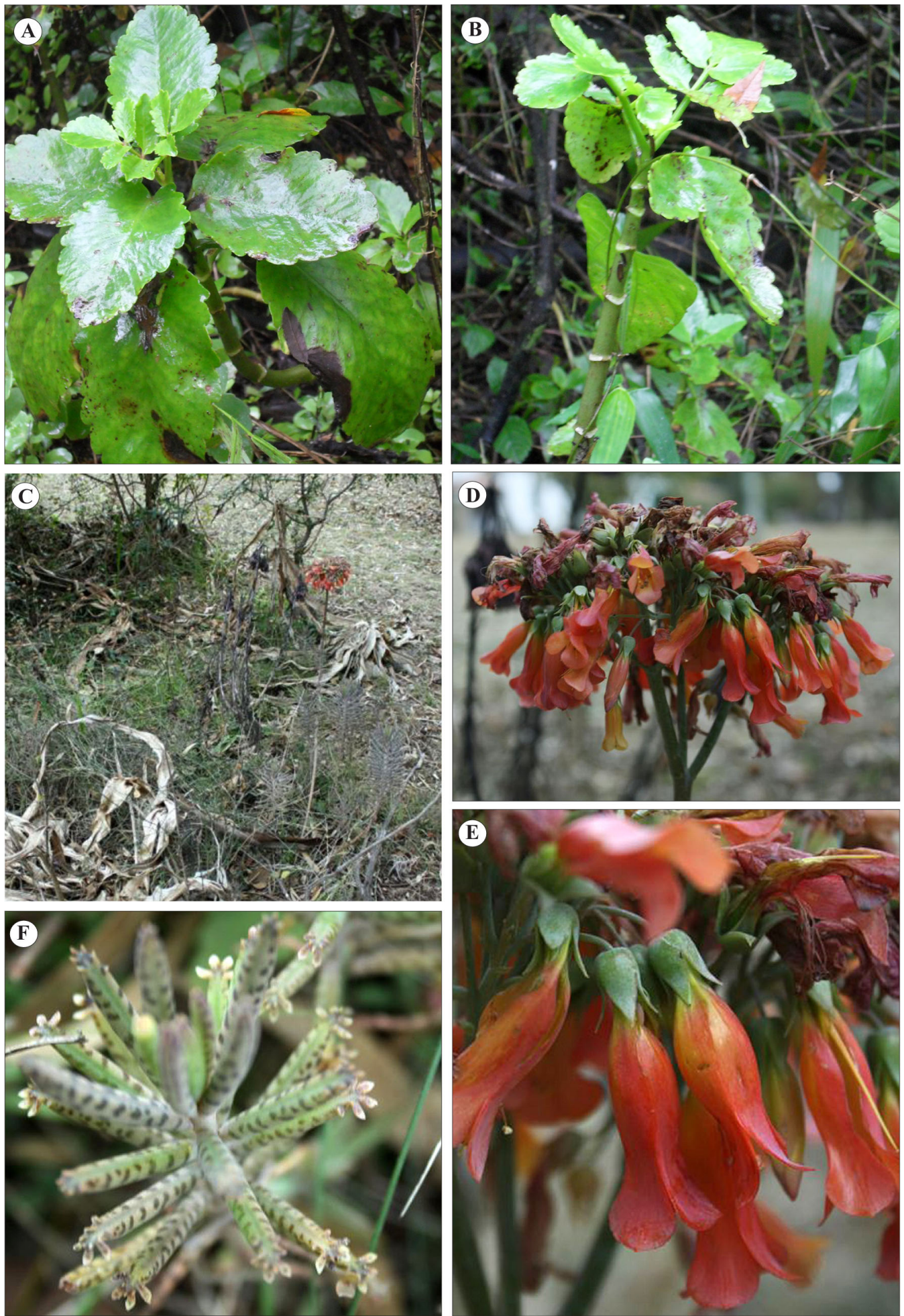

Fig. 3. Bryophyllum pinnatum. A-B: Plantas en Concepción de la Sierra, Misiones (Fotos: P.C. Stampella). Bryophyllum delagoense. C: Planta en el Palmar, Entre Ríos. D: Inflorescencia. E: Detalle de las flores. F: Detalle de las hojas cilíndricas con propágulos (Fotos: A. Maranta). 
2002). En Brasil, se emplea con fines litúrgicos (Pieres \& al., 2009). En Corrientes, las hojas se aplican como emplastos para eliminar los callos (Martínez Crovetto, 1981). En Misiones, según los informantes entrevistados, los flores son utilizadas por los niños de la misma manera que las de $B$. fedtschenkoi.

Estudios experimentales han permitido comprobar, en correlato con sus usos populares, su actividad inmunomoduladora (Cruz \& al., 2012), anticáncer y antiviral (Mahata \& al., 2012), hepatoprotectora (Yadav \& Dixit, 2003), neurosedante y miorrelajante (Yemitan \& Salahdeen, 2005), antiinflamatoria, analgésica y antidiabética (Ojewole, 2005; Afzal \& al., 2012), antimicrobiana y antioxidante (Tatsimo \& al., 2012).

Material examinado: ARGENTINA. Misiones. Concepción de la Sierra. Santa María, Paraje Cerro Mártires, chacra dos Santos, junto a $B$. fedtschenkoi, pero menos abundante, 18-VII2012, fl., Stampella 153 (LP). Corrientes. Capital. Ciudad de Corrientes, costas modificadas del río Paraná, 25-IX-1977, fl., Schinini 14270 (CTES).

4. Bryophyllum delagoense (Eckl. \& Zeyh.) Schinz, Mém. Herb. Boissier 10: 38. 1900. (Fig. 3 C-F).

Kalanchoe delagoensis Eckl. \& Zeyh., Enum. Pl. Afr. Austral. 305. 1837.

Bryophyllum tubiflorum Harv., Fl. Cap. 2: 380. 1862.

Kalanchoe verticillata Scott-Elliot, J. Linn. Soc., Bot. 29(197): 14-15, pl. 3. 1891.

Kalanchoe tubiflora (Harv.) Raym.-Hamet, Beih. Bot. Centralbl. 29(2): 41. 1912, nom. illeg.

Bryophyllum verticillatum (Scott-Elliot) A.Berger, Nat. Pflanzenfam. (ed. 2), 18a: 411. 1930.

Hierbas bienales o perennes, 0,3-2 $\mathrm{m}$ alt. Tallos robustos, erectos a procumbentes, teretes, no ramificados. Hojas opuestas en brotes jóvenes, ternadas o alternas en la planta adulta, simples, carnosas, sésiles o con pecíolo indistinguible; láminas cilíndricas, 3-15 cm long. $\times$ 0,3-0,6 cm lat., en general, verde grisáceas con manchas oscuras, margen entero, excepto por 2-9 dientes apicales cónicos, con propágulos en sus axilas. Cimas corimbiformes densas, $5-20 \mathrm{~cm}$ diám., ramas hasta $3 \mathrm{~cm}$ long.; pedicelos 0,5-3 cm long. Cáliz rojizo o verde pálido, con líneas rojizas; tubo 3-6 mm long.; lobos 5-10 mm long., agudos o acuminados. Corola 2,2-4 cm long., anaranjada a escarlata; lobos 6-12 $\mathrm{mm}$ long. $\times$ 6-9 mm lat., obtusos o apiculados. Estambres incluidos. Carpelos 5,5-6,5 mm long.; estilos hasta $20 \mathrm{~mm}$ long. Nectarios 0,7-2 mm long. Semillas 0,6-2,5 mm long. $2 n=68$ (Moran, 2009).

Distribución geográfica. Originaria de Madagascar, naturalizada en zonas cálidas, como el sur de Europa, Macaronesia, India, África, Hawái, Australia y Nueva Zelanda, sur de los Estados Unidos, México y Centroamérica, Antillas, Sudamérica septentrional, Perú, Bolivia, sur de Brasil. En Sudáfrica y Australia es maleza e invasora (Stevens \& al., 2001; Villaseñor \& EspinosaGarcía, 2004; Domingues \& Freitas, 2006; Pino Infante, 2006; Guillot Ortíz \& al., 2009; Moran, 2009; Walters \& al., 2011; Randall, 2012). En la Argentina se cultiva (Dimitri, 1987) y crece naturalizada en Entre Ríos (Parque Nacional El Palmar) y zonas urbanas de Buenos Aires (Avellaneda, Quilmes, La Plata).

Nombres vernáculos: "calanchoe", “candelabro", "madre de millones".

Etnobotánica. Ornamental; se multiplica rápidamente por brotes basales, propágulos foliares y trozos de hojas que enraízan fácilmente. Es tóxica para el ganado y los seres humanos (Kellerman \& al., 2005; Guillot Ortíz \& al., 2009; Walters \& al., 2011).

Material examinado: ARGENTINA. Entre Ríos. Colón. Parque Nacional El Palmar, parque de la Intendencia, 4-XI-2008, fl., Delucchi 3416 (LP). Buenos Aires. Avellaneda. Wilde, Avenida Onsari nro. 422, en balcón, 24-IX-2012, fl., Guerrero 207 (LP). Quilmes. Bernal, Ramella, entre Roca y Carabelas, 29-IX-2012, fl., Guerrero 236 (LP); ibídem, 29-IX-2012, fl., Guerrero 238 (LP). La Plata, Villa Montoro, calle 1 (95 y 96), 29-X-2012, fl., Guerrero 207 (LP). 


\section{Discusión y conclusiones}

Las especies tratadas constituyen un ejemplo de naturalización de especies exóticas introducidas con fines ornamentales, proceso en el que juegan un rol fundamental los jardines y huertos familiares, que ofician de centros de dispersión. La dinámica de la naturalización de especies exóticas es un tema central en diversos estudios en todo el mundo, dado que las especies naturalizadas son potenciales invasoras o malezas. Las especies naturalizadas se dispersan por sus propios medios y se integran a la flora local, sin intervención humana. Son invasoras (o malezas, en el caso de los cultivos), las especies naturalizadas cuya expansión compromete la biodiversidad local (Richardson \& al., 2000; Rejmánek, 2000; Pyšek \& al., 2004; Pyšek \& Richardson, 2006).

En este contexto, las ornamentales naturalizadas constituyen un grupo de especial interés (Mulvaney, 1991; Marco \& al., 1999; Kowarik, 2005; Dehnen-Schmutz \& Touza, 2008). Para la Argentina, la presente contribución representa un aporte a ese campo, junto a trabajos anteriores en la misma línea (Delucchi \& Keller, 2010; Delucchi, 2011; Hurrell \& al., 2011, 2012; Keller \& al., 2012).

Las especies de Bryophyllum tratadas se expanden y naturalizan principalmente por vía vegetativa. Sus propágulos foliares, hojas caídas o trozos de las mismas con propágulos, crecen y se desarrollan en sitios modificados, bordes de caminos, capueras, barrancas, roquedales, espacios rellenados con escombros y desechos de jardines; también, sobre techos en áreas urbanas. Los propágulos enraízan rápidamente, incluso sobre sustratos muy ligeros, y del mismo modo que ocurre en los jardines, forman colonias densas en torno a las plantas originales, por lo cual han recibido los nombres de "madre de miles" o "madre de millones", en diversos países.

En su naturalización, las plantas se expanden primero dentro de los jardines; luego en sus alrededores inmediatos, y se extienden más tarde en bordes de caminos; si los predios han sido abandonados, perduran como ergasiolipófitas; posteriormente, se integran a las comunidades locales, como es el caso de $B$. fedtschenkoi y B. pinnatum en Concepción de la Sierra, Misiones. Si sumamos el dato etnobotánico sobre el empleo lúdico de estas plantas, que los niños resignifican como propias "del monte" ("locales"), la integración es también cultural. En Corrientes, la cita de B. pinnatum en la costa del Paraná (Martínez Crovetto, 1981), sugiere un proceso de naturalización similar.

B. daigremontianum en Misiones, y B. fedtschenkoi en Salta, se hallan en el borde de rutas, en sitios con mucha pendiente, por lo común rocosos y con escaso sustrato. En estos lugares son frecuentes los accidentes vehiculares fatales y se levantan cruces para recordar a las personas fallecidas. En general, esas cruces son adornadas con distintas plantas, entre las cuales se incluyen estas especies, elegidas por su rusticidad; luego, con facilidad, se expanden y colonizan los alrededores.

Las cuatro especies tratadas se extienden en los espacios urbanos de la Capital Federal, Avellaneda, Quilmes y La Plata, en terrenos baldíos, escombros, bordes de vías férreas, balcones, terrenos y canteros abandonados. $B$. daigremontianum y B. fedtschenkoi, asimismo, se expanden sobre toldos metálicos, aleros de cemento y techos, donde enraízan fácilmente sobre un sustrato escasísimo. B. fedtschenkoi ha sido hallada, además, sobre el ramaje de árboles urbanos, en iguales condiciones de sustrato.

\section{Agradecimientos}

Al personal de los herbarios CTES, LP y BAA (Facultad de Agronomía, Universidad de Buenos Aires) y de la biblioteca y herbario SI (Instituto de Botánica Darwinion, San Isidro); a los revisores anónimos, por sus sugerencias; a los informantes entrevistados, en especial, a la familia Amarilla-dos Santos (Paraje Cerro Mártires, Misiones); a Aristóbulo Maranta, del Parque Nacional El Palmar, por su gentil colaboración; a Pamela Carrión y Juan José Vattimo por su cooperación en las tareas de herborización, y a Liliana Sancho y Martha Freire por facilitarnos dicho trabajo. 


\section{Bibliografía}

AFZAL, M., G. GUPTA, I. KAZMI, M. RAHMAN, O. AFZAL, J. ALAM, K. HAKEEM, M. PRAVEZ, R. GUPTA \& F. ANWAR. 2012. Anti-inflammatory and analgesic potential of a novel steroidal derivative from Bryophyllum pinnatum. Fitoterapia 83(5):853858.

ALBUQUERQUE, U. P. \& R. F. LUCENA. 2004. Métodos e técnicas na pesquisa etnobotânica. 190 pp. Livro Rápido/NUPEEA, Recife.

ARANGO CARO, S. 2004. Ethnobotanical studies in the Central Andes (Colombia): Knowledge distribution of plant use according to informant's characteristics. Lyonia 7(2): 89-104.

BARUKIAL, J. \& J.N. SARMAH. 2011. Ethnomedicinal plants used by the people of Golaghat District, Assam, India. Int. J. Med. Arom. Plants 1(3): 203-221.

BISWAS, S., A. CHOWDHURY, J. DAS, S.M. ZAHID HOSEN, R. UDDIN \& M. S. RAHAMAN. 2011. Literature review on pharmacological potentials of Kalanchoe pinnata (Crassulaceae). Afr. J. Pharm. Pharmacol. 5(10): 1258-1262.

CHAGASNOGUEIRA, M., S.A. BEZERRA, F.MACÊDO NUNES \& L.C. MAFFEI. 2012. Ethnobotanical study in the community of Riacho das Vacas (City of Caetité, Bahia, Brazil) reveals the local population's knowledge about medicinal plants and their uses. Wudpecker J. Agric. Res. 1(8): 302-316.

CHERNETSKYY, M. A. 2011. Problems in Nomenclature and Systematics in the Subfamily Kalanchoideae (Crassulaceae) over the years. Acta Agrobot. 64: 67-74.

- 2012. The role of morpho-anatomical traits of the leaves in the taxonomy of Kalanchoideae (Crassulaceae). Modern Phytomorphol. 1: 15-18.

CRUZ, E.A., S. REUTER, H. MARTIN, N. DEHZAD, M. MUZITANO, S.S. COSTA, B. ROSSI-BERGMANN, R. BUHL, M. STASSEN \& C. TAUBE. 2012. Kalanchoe pinnata inhibits mast cell activation and prevents allergic airway disease. Phytomedicine 19(2): 115-121.

DEHNEN-SCHMUTZ, K. \& J. TOUZA. 2008. Plant invasions and ornamental horticulture: pathway, propagule pressure and the legal framework. En: J.A. Teixeira da Silva (Ed.), Floriculture, Ornamental and Plant Biotechnology. Vol. 5: 15-21. Global Sci. Books. London.

DELUCCHI, G. 2011. Sinopsis de las especies adventicias de Rosaceae: Subfamilia Prunoideae. Bonplandia 20(1): 73-94.

\& H. KELLER. 2010. La naturalización del "níspero", Eriobotrya japonica (Rosaceae, Maloideae), en la Argentina. Bonplandia 19(1): 71-77.

DIMITRI, M. J. 1987. Crasuláceas. Encicl. Argent. Agric.
Jard. (ed. 3) I (1): 422-429. Acme, Buenos Aires.

DOMINGUES J. \& H. FREITAS. 2006. Exotic naturalized flora of continental Portugal. A reassessment. Bot. Complutensis 30: 117-130.

EGGLI, U. 2003. Illustrated handbook of succulent plants.VI. Crassulaceae. 458 pp. Springer, Berlin.

ESTRADA, E., J.A. VILLARREAL, C. CANTÚ, I. CABRAL, L. SCOTT \& C. YEN. 2007. Ethnobotany in the Cumbres de Monterrey National Park, Nuevo León, México. J. Ethnobiol. Ethnomed. 3: 8, doi:10.1186/1746-4269-3-8.

FREIRE-FIERRO, A. 2008. Crassulaceae. En Zuloaga, F. O., O. Morrone \& M. J. Belgrano (Eds.). Catálogo de las Plantas Vasculares del Cono Sur. Monographs of Systematic Botany 107. Missouri Botanical Garden Press, St. Louis.

FU K. \& M. G. GILBERT. 2001. Bryophyllum. En Wu, Z. Y. \& P. H. Raven (eds.), Fl. of China 8: 204. Science Press, Beijing - Missouri Bot. Gard. Press, St. Louis

GARCÉS, H. \& N. SINHA. 2009. The 'mother of thousands' (Kalanchoë daigremontiana): a plant model for asexual reproduction and CAM studies. Cold Spring Harb. Protoc. 2009 (10), doi: 10.1101/ pdb.emo133.

- C. CHAMPAGNE, B.T. TOWNSLEY, S. PARK, R. MALHO, M.C. PEDROSO, J.J. HARADA \& N. SINHA. 2007. Evolution of asexual reproduction in leaves of the genus Kalanchoë. Proc. Natl. Acad. Sci. U.S.A. 104(39): 15578-15583.

GHASI, S., C. EGWUIBE, P. ACHUKWU \& J.C. ONYEANUSI. 2011. Assessment of the medical benefit in the folkloric use of Bryophyllum pinnatum leaf among the Igbos of Nigeria for the treatment of hypertension. Afr. J. Pharm. Pharmacol. 5(1): 83-92.

GODÍNEZ-CARABALLO, D. \& G. VOLPATO. 2008. Plantas medicinales que se venden en el mercado El Río, Camagüey, Cuba. Rev. Mex. Biodiv. 79: 243-259.

GUILLOT ORTÍZ, D., E. LAGUNA LUMBRERAS, J.A. ROSELLÓ PICORNELL. 2009. La familia Crassulaceae en la flora alóctona valenciana. Bouteloua Monogr. 4: 1-106.

HURRELL, J. A., P. CABANILLAS \& G. DELUCCHI. 2011. Wisteria sinensis (Leguminosae) adventicia en la Argentina. Primer registro y mecanismos de expansión. Revista Mus. Argent. Ci. Nat., n.s. 13(2): 125-130.

- P. CABANILLAS, F. BUET COSTANTINO \& G. DELUCCHI. 2012. Bignoniaceae adventicias en la Argentina. Primera cita de Podranea ricasoliana y nuevos registros de Campsis radicans. Revista Mus. Argent. Ci. Nat., n.s. 14(1): 15-22.

JIOFACK, T., C. FOKUNANG, N. GUEDJE, V. KEMEUZE, E. FOnGNZOSSIE, B. A. NKONGMENECK, P. M. MAPONGMETSEM \& N. TSABANG. 2010. Ethnobotanical uses of 
medicinal plants of two ethnoecological regions of Cameroon. Int. J. Med. Medical Sci. 2(3): 60-79.

KELLER, H.A., J.A. HURRELL, R.O. VANNI \& G. DELUCCHI. 2012. Senna macranthera (Leguminosae), una especie ornamental naturalizada en la Argentina. Bonplandia 21(1): 55-60.

KELLERMAN, T.S., J.A. COETZER, T.W. NAUDÉ \& C. J. BOTHA. 2005. Plant poisonings and mycotoxicoses of livestock in Southern Africa. Ed. 2, 256 pp. Oxford University Press, Cape Town.

KOWARIK, I. 2005. Urban ornamentals escaped from cultivation. En: J. Gressel (Ed.), Crop ferality and volunteerism, pp. 97-121. CRCPress, Boca Raton.

LANE, R. 1997. Oral histories and scientific knowledge in understanding environmental change: a case study in the Tumut Region, New South Wales, Australia. Geographical Res. 35 (2): 195-205.

MAHATA, S., S. MARU, S. SHUKLA, A. PANDEY, G. MUGESH, B.C. DAS \& A.C. BHARTI. 2012. Anticancer property of Bryophyllum pinnata leaf on human cervical cancer cells. BMC Complement. Altern. Med. 12:15, doi:10.1186/1472-6882-12-15.

MARCO, A.S. LAVERGNE, T. DUTOIT \& V. BERTAUDIERE-MONTES. 1999. From the backyard to the backcountry: how ecological and biological traits explain the escape of garden plants into Mediterranean old fields. Biological Invasions 12(4): 761-779.

MARTIN, G.J. 2004. Ethnobotany. A methods manual. 268 pp. Earthscan, London.

MARTÍNEZ CROVETTO, R. 1981. Las plantas utilizadas en medicina popular en el noroeste de Corrientes (República Argentina). Miscelánea 69: 7-140. Fund. Miguel Lillo, Tucumán.

MORAN, R. V. 2009. Bryophyllum. En F1. of North America Edit. Committee (eds.), Fl. of North America North of Mexico 8: 158-161. Oxford Univ. Press, New York.

MULVANEY, M.J. 1991. Far from the garden path: an identikit picture of woody ornamental plants invading south-eastern Australian bushland. PhDThesis. Australian National University, Canberra.

NAHAR, K., M. G. U. KHAN, M. S. RAHMAN, B. BEGUM \& M.A. RASHID. 2008. Antimicrobial and cytotoxic activities of Bryophyllum daigremontianum. Dhaka Univ. J. Pharm. Sci. 7(1): 99-101.

OJEWOLE, J.A. 2005. Antinociceptive, anti-inflammatory and antidiabetic effects of Bryophyllum pinnatum leaf aqueous extract. J. Ethnopharmacol. 99(1): 13-19.

OLOWOKUDEJO, J.D., A.B. KADIRI \& V.A. TRAVIH. 2008. An Ethnobotanical Survey of Herbal Markets and Medicinal Plants in Lagos State of Nigeria. Ethnobot. Leafl. 12: 851-865.

PARDO, O. 2002. Etnobotánica de algunas cactáceas y suculentas del Perú. Chloris chilensis 5: 1. Disponible: $<$ http://www.chlorischile.cl/> [Consulta: 16-X2012].
PARSLEY, L.C. \& A.E. RUSHING. 2003. The sequence of development of leaf propagules in Bryophyllum daigremontianum. Botany 2003, Alabama.

PIERES, M.V. P.P. ABREU, C. SILVA SOARES, B. SOUZA1, D. MARIANO, D. DA COSTA SILVA \& E.A. ROCHA1. 2009. Etnobotânica de terreiros de candomblé nos municipios de Ilhéus e Itabuna, Bahia, Brasil. Rev. Bras. Bioci. (Porto Alegre) 7(1): 3-8.

PINO INFANTE, G. 2006. Estado actual de las suculentas en el Perú. Revista Zonas Áridas 10: 155-173.

PYŠEK, P. \& D. RICHARDSON. 2006. The biogeography of naturalization in alien plants. J. Biogeography 12: 2040-2050. , D. RICHARDSON, M. REJMÁNEK, G. WEBSTER, M. WILLIAMSON \& J. KIRSCHNER. 2004. Alien plants in checklists and floras: towards better communication between taxonomists and ecologists. Taxon 53(1): 131-143.

RANDALL, R. 2012. A Global Compendium of Weeds. Ed. 2. Dep. Agr. Food, Perth.

REJMÁNEK, M. 2000. Invasive plants: approaches and predictions. Austral Ecology 25: 497-506.

RICHARDSON, D. M., P. PYŠEK, M. REJMÁNEK, M. G. BARBOUR, F. DANE PANETTA \& C. J. WEST. 2000. Naturalization and invasion of alien plants: concepts and definitions. Diversity Distrib. 6: 93-107.

SARWAR, G.R. 2004. Crassulaceae. En Nasir, E. \& S. I. Ali (eds), Fl. Pakistan, vol. 209. Univ. of Karachi, Karachi.

STEVENS, W.D., C. ULlOA ULlOA, A. POOL \& O. MONTIEL (eds.). 2001. Flora de Nicaragua. Monogr. Syst. Bot. Missouri Bot. Dard. 85: 1-2666.

TATSIMO, S.J., J. de D. TAMOKOU, L. HAVYARIMANA, D. CSUPOR, P. FORGO, J. HOHMANN, J. R. KUIATE \& P. TANE. 2012. Antimicrobial and antioxidant activity of kaempferol rhamnoside derivatives from Bryophyllum pinnatum. BMC Res. Notes. 5: 158.

TENE, V., O. MALAGON, P. VITA, G. VIDARI, C. ARMiJOS \& T. ZARAGOZA. 2007. An ethnobotanical survey of medicinal plants used in Loja and Zamora-Chinchipe, Ecuador. J. Ethnopharmacol. 111: 63-81.

THIEDE, J. \& U. EGGLI. 2007. Crassulaceae. En Kubitzki, K. (ed.), The Families and genera of Vascular Plants. IX: 83-118. Springer, Berlin.

VEGA, M. 2001. Etnobotánica de la Amazonia peruana. 166 pp. Ed. Abya-Yala, Quito.

VILLASEÑOR, J. L. \& F. J. ESPINOSA-GARCÍA. 2004. The alien flowering plants of Mexico. Diversity and Distributions 10: 113-123.

WALTERS, M., E. FIGUEIREDO, N.R.CROUCH, P.J.D. WINTER, G. F. SMITH, H.G. ZIMMERMANN \& B.K. MASHOPE. 2011. Naturalised and invasive 
succulents of Southern Africa. Abc Taxa Vol. 11: i-x, 360 pp.

WHISTLER, W.A. 1988. Ethnobotany of Tokelau: the plants, their Tokelau names, and their uses. Econ. Bot. 42(2): 155-176.

YADAV, N.P. \& V.K. DIXIT. 2003. Hepatoprotective activity of leaves of Kalanchoe pinnata. J. Ethnopharmacol. 86(2-3): 197-202.

YEMITAN, O. \& H. SALAHDEEN. 2005. Neurosedative and muscle relaxant activities of aqueous extract of Bryophyllum pinnatum. Fitoterapia 76: 187-193.

Original recibido el 22 de octubre de 2012; aceptado el 3 de diciembre de 2012. 
